\title{
Spaceflight-induced Cardiovascular Changes and Recovery during NASA's Functional Task Test
}

N. M. Arzeno, ${ }^{1}$ M. B. Stenger, ${ }^{1}$ J. J. Bloomberg, ${ }^{2}$ and S. H. Platts ${ }^{2}$

${ }^{1}$ Wyle Integrated Science and Engineering Group, Houston, TX, and ${ }^{2}$ NASA, Johnson Space Center, Houston, TX

Microgravity-induced physiological changes could impair a crewmember's performance upon return to a gravity environment. The Functional Task Test (FTT) is designed to correlate these physiological changes to performance in mission-critical tasks. The Recovery from Fall/Stand Test (RFST) simulates one such task, measuring the ability to recover from a prone position and the cardiovascular response to orthostasis. The purpose of this study was to evaluate spaceflight-induced cardiovascular changes during the FTT. METHODS: Five astronauts participated in the FTT before 10-15 day missions, on landing day $(\mathrm{R}+0)$, and one $(\mathrm{R}+1)$, six $(\mathrm{R}+6)$ and thirty $(\mathrm{R}+30)$ days after landing. The RFST consisted of a 2-minute prone rest followed by a 3-minute stand during which heart rate (HR, Holter) and continuous blood pressure (BP, Finometer) were measured. Spectral heart rate variability (HRV) was calculated during the RFST to approximate autonomic function. Statistical analysis was performed with two-factor repeated measures ANOVA. RESULTS: During RFST, HR was higher on $\mathrm{R}+0$ than preflight $(\mathrm{p}<0.004)$. This increase in HR persisted on $\mathrm{R}+1$ and $\mathrm{R}+6$ during the stand portion of RFST $(\mathrm{p}<0.026)$. BP was well-regulated on all test days. Parasympathetic activity was diminished on $\mathrm{R}+0(\mathrm{p}=0.035)$. Sympathovagal balance tended to be affected by spaceflight (main effect, $\mathrm{p}=0.072$ ), appearing to be slightly elevated during postflight RFST except on $\mathrm{R}+30$. Additionally, analysis of HR during the functional tasks yielded a higher HR on R+0 than preflight during 8 of 11 tasks analyzed, where all tasks had HR return to preflight values by $\mathrm{R}+30(\mathrm{p}<0.05)$. CONCLUSION: Spaceflight causes an increase in HR, decrease in parasympathetic activity, and increase in sympathovagal balance, which we confirmed during RFST. These spaceflight-induced changes seen in the RFST, along with the increased postflight HR in most functional tasks, can be used to assess functional performance after short-duration spaceflight. 